\title{
Whole-exome sequencing identifies somatic mutations and intratumor heterogeneity in inflammatory breast cancer
}

Rui Luo ${ }^{1,5}$, Weelic Chong $\mathbb{D D}^{1,5}$, Qiang Wei ${ }^{2}$, Zhenchao Zhang ${ }^{1}$, Chun Wang ${ }^{1}$, Zhong Ye ${ }^{1}$, Maysa M. Abu-Khalaf ${ }^{1}$, Daniel P. Silver ${ }^{1}$, Robert T. Stapp ${ }^{3}$, Wei Jiang ${ }^{3}$, Ronald E. Myers ${ }^{1}$, Bingshan Li $^{2}$, Massimo Cristofanilli ${ }^{4}$ and Hushan Yang (iD) ${ }^{1 凶}$

Inflammatory breast cancer (IBC) is the most aggressive form of breast cancer. Although it is a rare subtype, IBC is responsible for roughly $10 \%$ of breast cancer deaths. In order to obtain a better understanding of the genomic landscape and intratumor heterogeneity (ITH) in IBC, we conducted whole-exome sequencing of 16 tissue samples (12 tumor and four normal samples) from six hormone-receptor-positive IBC patients, analyzed somatic mutations and copy number aberrations, and inferred subclonal structures to demonstrate ITH. Our results showed that KMT2C was the most frequently mutated gene $(42 \%, 5 / 12$ samples), followed by HECTD1, LAMA3, FLG2, UGT2B4, STK33, BRCA2, ACP4, PIK3CA, and DNAH8 (all nine genes tied at 33\% frequency, 4/ 12 samples). Our data indicated that PTEN and FBXW7 mutations may be considered driver gene mutations for IBC. We identified various subclonal structures and different levels of ITH between IBC patients, and mutations in the genes EIF4G3, IL12RB2, and PDE4B may potentially generate ITH in IBC

npj Breast Cancer (2021)7:72; https://doi.org/10.1038/s41523-021-00278-w

\section{INTRODUCTION}

Inflammatory breast cancer (IBC) is an aggressive form of breast cancer defined by the rapid onset of inflammatory signs (such as erythema, edema, warmth, and induration) involving more than one-third of the breast ${ }^{1-3}$. IBC accounts for $1-6 \%$ of breast cancer cases $^{2,4,5}$ yet causes roughly $10 \%$ of breast cancer deaths $s^{6,7}$. The prognosis in patients with IBC is worse than in non-IBC, with the 3year survival rate for IBC patients far lower (around 40\%) than patients with other types of breast carcinoma (around $85 \%)^{5,8}$. Although treatment approaches based on hormone-receptor (HR) or HER2 status are available, there are no treatments that are specifically recommended for tumors with an IBC phenotype. The scarcity of data from IBC patients and the poor understanding at the molecular level has hindered the development of specific therapeutic interventions. In order to develop potential IBCspecific targeted therapies, obtaining more genomic information is crucial.

Intratumor heterogeneity (ITH) arises from heritable and stochastic genetic and epigenetic changes, as well as environmental variations within the tumor' ${ }^{9}$. Since tumors with ITH have subclones with distinct mutations that may relate to cancerspecific phenotypes, ITH is intricately related to cancer progression, resistance to therapy, and recurrences ${ }^{10}$. It is clear that a better understanding of ITH is very important to the development of genome-informed precision medicine ${ }^{11}$.

The rapidly evolving technology of next-generation sequencing (NGS) has made it possible to analyze genomic characteristics of tumor samples at an unprecedented speed. Since 2015, eight NGS-based studies on IBC tumors have been published. Among them, six out of eight used targeted sequencing ${ }^{12-18}$, and two conducted whole-exome sequencing $(\mathrm{WES})^{19,20}$. These studies reported frequently mutated genes in IBC, such as TP53 (43-75\%), PIK3CA (13-42\%), BRCA2 (13-26\%), ARID1A (10-21\%), RB1
$(11-16 \%)$, and PTEN $(11-15 \%)^{12-18}$. Frequent HER3 hotspot mutations were also found in IBC tumors and cell line studies confirmed a role for mutant $H E R 3$ in IBC cell proliferation ${ }^{15}$. Frequent genomic alterations in the PI3K/AKT/mTOR pathway have been seen ${ }^{15}$, and somatic activation of this pathway (i.e., PIK3CA activating mutation or gain ${ }^{14}$, ERBB2 activating mutation, PTEN deletion, AKT1 activating mutation) was significantly associated with shorter progression-free survival (PFS) in trastuzumab-naïve HER2-positive IBC patients ${ }^{19}$. However, most of these studies were based on targeted sequencing panels, and none of them provided information for intratumor subclonal structures or evaluated ITH.

In the current study, we performed whole-exome sequencing in 16 tissue samples (12 tumor and 4 normal samples) from six IBC patients to obtain a comprehensive understanding of the IBC genomic landscape. Based on the mutation calls and somatic copy number alterations, we characterized ITH and subclonal structures, identified primary and secondary driver genes for the tumor and subclone formation, which could shed light on potential new treatment strategies for IBC.

\section{RESULTS}

\section{Patient and sample description}

Clinical and pathological information of the six IBC patients (P1-P6) are provided in Supplementary Table 1. The median age at sample collection time was 56 years (ranging from 36 to 72 years). All six patients had $\mathrm{HR}+$ tumors, with $5 / 6$ (83.3\%) patients having estrogen-receptor-positive $(E R+)$ tumors, and the other had a progesterone-receptor positive $(\mathrm{PR}+)$ tumor. By only considering $\mathrm{HR}+\mathrm{IBC}$ tumors, our study eliminated additional confounding introduced by differences in HR subtypes seen in previous studies. Details of the tumor and normal tissue samples

\footnotetext{
${ }^{1}$ Department of Medical Oncology, Sidney Kimmel Cancer Center, Thomas Jefferson University, Philadelphia, PA, USA. ²Department of Molecular Physiology and Biophysics, Vanderbilt University, Nashville, TN, USA. ${ }^{3}$ Department of Pathology, Sidney Kimmel Cancer Center, Thomas Jefferson University, Philadelphia, PA, USA. ${ }^{4}$ Division of Hematology Oncology, Feinberg School of Medicine, Northwestern University, Chicago, IL, USA. ${ }^{5}$ These authors contributed equally: Rui Luo, Weelic Chong. ${ }^{\circledR}$ email: hushan.yang@jefferson. edu
} 


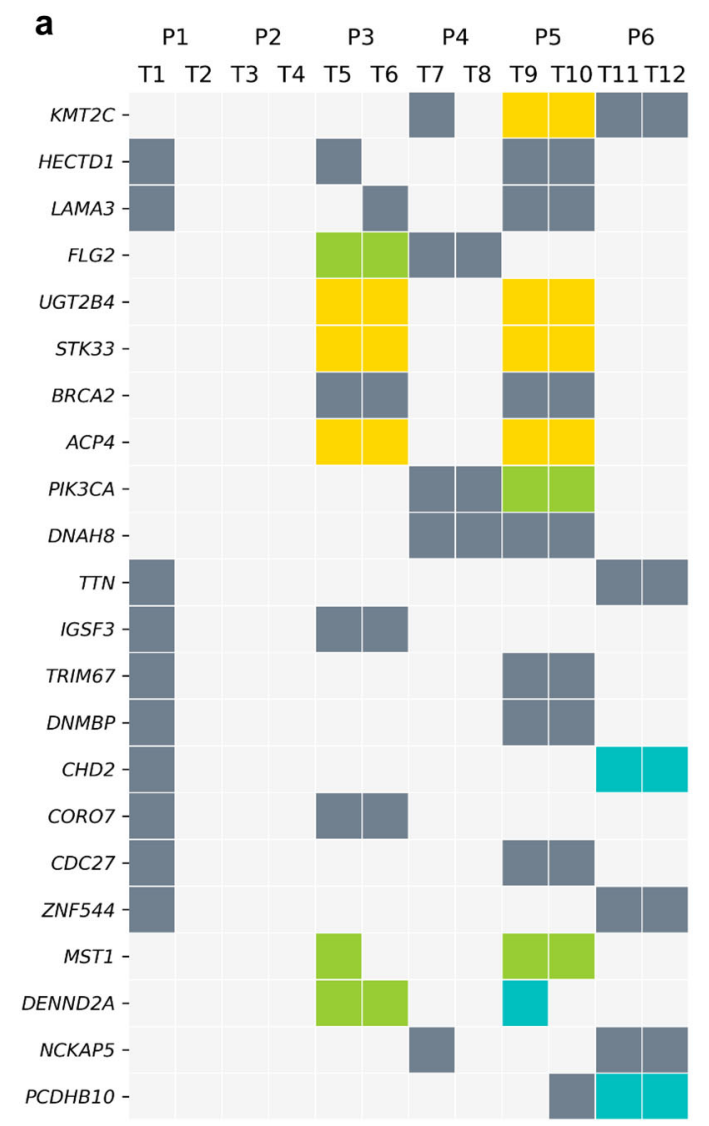

b

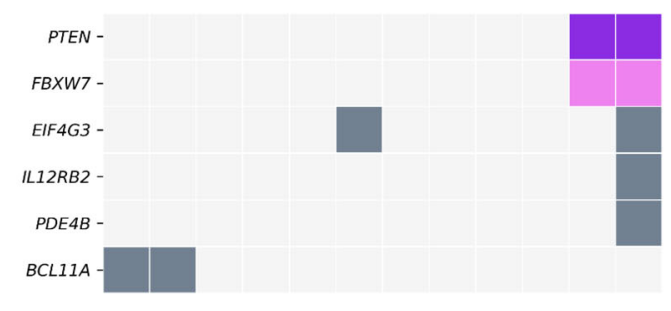

nonsynonymous SNV

nonframeshift substitution

nonframeshift deletion

stopgain

splicing

frameshift deletion

Fig. 1 Somatic mutation profile for 12 tumor samples of six patients. a The most frequently mutated genes are shown in a heatmap, with the columns representing the 12 tumor samples from six patients, and the rows representing genes. The different colors indicate the type of mutation as indicated in the figure. b Heatmap of mutations in selected genes known to be involved in cancer pathogenesis or progression.

obtained from the six IBC patients are found in Supplementary Table 2. The samples from P2 were obtained from an incisional biopsy, which limited the volume of tissue obtained, and these samples were subsequently found to be insufficient for conducting subclone identification.

\section{Sequencing quality validation}

We achieved a mean sequencing depth of $\sim 170 \times$ (ranging from 133 to $210 \times$, Supplementary Table 3), with mapping rates exceeding $99 \%$ in all 16 samples. After stringent filtering criteria (see Methods), we obtained a total of 1477 somatic mutations. We called 293, 15, 261, 120, 495, and 293 somatic mutations, respectively, in patients P1-P6 (Supplementary Data 1). Four of the six patients (P1, P2, P4, and P6) had matched normal samples, allowing us to validate the stringency of our mutation calling pipeline (see Methods). We identified artifactual mutations in one, six, one, and four instances, respectively, in patients P1, P2, P4, and P6. Artifactual mutations in normal samples also had much lower allele frequencies (AFs) and tended to be obtained at lower depths compared to tumor mutation calls, which indicated that FFPE-induced artifacts had negligible effects to the data presented in our study (Supplementary Fig. 1).

\section{Somatic mutation identification}

We used a somatic mutation classification system as previously described $^{21}$. Five of six patients exhibited mutational signatures characterized predominantly by $\mathrm{C}>\mathrm{T}$ transitions, with the sixth patient P6 showing a mix of $C>G$ and $C>T$ transitions (Supplementary Fig. 2). These results were consistent with previous reports for breast cancer, which have also found $C>T$ transitions to constitute the majority of somatic mutations ${ }^{21,22}$.

In total, we found 787 mutated genes from the 12 tumor samples in six patients. In these samples, KMT2C was the most frequently mutated gene (5/12 samples, 42\%). Nine mutated genes were found in four samples (33\%, including HECTD1, LAMA3, FLG2, UGT2B4, STK33, BRCA2, ACP4, PIK3CA, and DNAH8), and 12 genes were mutated in three different samples $(25 \%$, including TTN, IGSF3, TRIM67, DNMBP, CHD2, CORO7, CDC27, ZNF544, MST1, DENND2A, NCKAP5, and PCDHB10). Figure 1a shows the 22 most frequently mutated genes. In addition, mutations in 244 genes were found in two tumor samples, with the remaining gene mutations (in 521 genes) private to single tumor samples.

We also analyzed the gene mutations at the patient-level. $K M T 2 C, H E C T D 1$, and LAMA3 were the most frequently mutated genes as they were shared by three of six patients (50\%). Histone methyltransferase $K M T 2 C$ is a tumor suppressor gene reported to be a driver gene for breast cancer ${ }^{23,24}$. There were 57 mutated genes identified within two patients $(2 / 6,33 \%)$, and the rest of the mutated genes were not common to multiple patients. All counted mutations were nonsynonymous (i.e., frameshift/nonframeshift indel, stop-gain/stop-loss, splicing, or nonsynonymous SNV).

\section{Copy number aberration (CNA) inference}

We obtained $\sim 25,000$ germline variants in each patient with matched normal samples (P1, P2, P4, and P6). We used TITAN, a probabilistic model that simultaneously infers CNA and loss of heterozygosity $(\mathrm{LOH})$ segments from read depth and digital allele ratios at germline heterozygous SNP loci across the exome from 

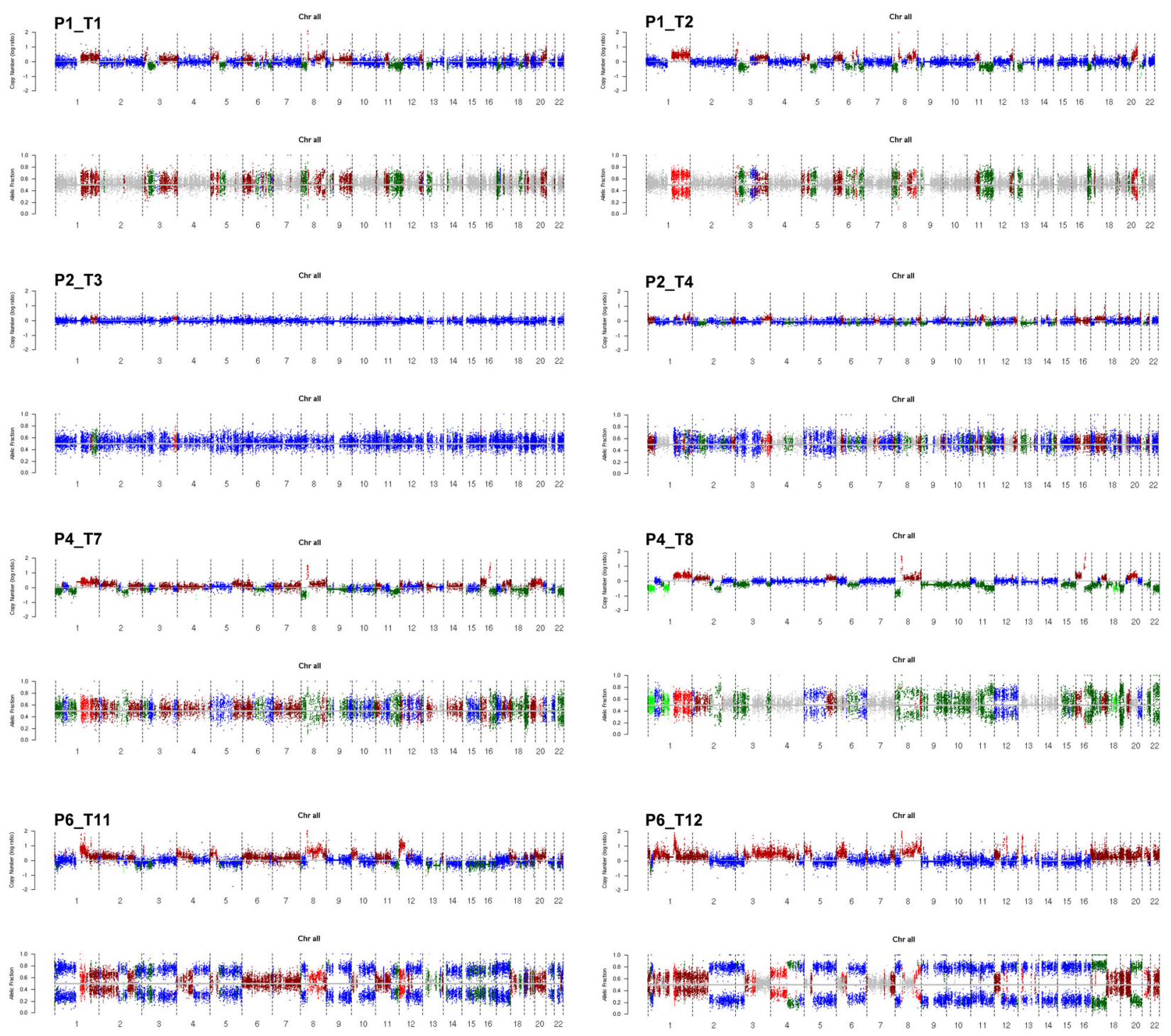

Fig. 2 Graphical representations of copy number alterations (CNAs) and allelic fractions (AFs) from two tumors per patient. Two tumors are obtained from each patient, represented on the left and right of the figure. Data from four patients with matched normal samples are available, and four sets of CNAs and AFs plots are presented. Within each set, the CNA plot is shown above the AF plot. For the CNA plot, the $y$-axis is the log2 ratio of the copy numbers of tumor to normal sample, both normalized for read depth. Points close to 0 (midline) represent no change in copy number, above 0 are copy gains, and below 0 are deletions. Regions are colored as follows: bright green (homozygous deletion), green (hemizygous deletion), blue (diploid heterozygous or copy-neutral loss of heterozygosity), dark red (copy number gain), and red (allele-specific CNA, unbalanced CNA, balanced CNA). The $x$-axis represents chromosomes. For the AF plot, the $y$-axis is the frequency of the reference allele in a germline heterozygous SNV, and the expected heterozygous frequency of 0.5 is the midline. Data points close to 1 represent homozygous reference, and data points close to 0 represent the homozygous nonreference base. Regions are colored as follows: gray (heterozygous, or balanced CNA), bright green (homozygous deletion), green (hemizygous deletion), blue (copy-neutral loss of heterozygosity), dark red (copy number gain), and red (allele-specific CNA, unbalanced CNA). The $x$-axis represents chromosomes.

tumor WES data ${ }^{25}$. Figure 2 shows the profiles of CNAs for the four patients with matched normal samples. We observed that patient P2 had a relatively low tumor cell fraction. Patient P6 had the best sample quality and showed extensive LOH.

\section{Subclone identification}

Using CNA information, we conducted PyClone analysis to estimate cancer cell fractions (CCFs) of all mutations and then assigned each mutation to different subclones (see Methods). For each patient, we obtained the subclone CCF density (represented as violin plots) and plotted CCFs in one tumor sample against the other tumor sample (as a scatter plot) (Fig. 3). Major subclones from the density plots are labelled in the same color in the scatter plot.
For patient P6 (Fig. 3a, b), we observed six distinct subclones with different cluster CCFs. Subclone 4, 5, and 11 all had very low subclone CCFs in one of the two samples (but high CCFs in the other sample), indicating clear ITH. Subclone 9 had cluster CCFs of greater than 0.7 in both samples, suggesting a high possibility of this subclone containing driver genes. This subclone also contained mutations in PTEN and FBXW7, both tumor suppressor genes previously reported ${ }^{26,27}$ as driver genes for breast cancer. Subclone 11 contained EIF4G3, IL12RB2, and PDE4B mutations, and all three mutations had zero allele frequencies in the tumor sample P6_T11, indicating the possibility of secondary driver genes for this subclone. We used Integrative Genomics Viewer (IGV) to check and confirm that the high CCFs of these genes were not caused by duplication. EIF4G3, IL12RB2, and PDE4B genes are 


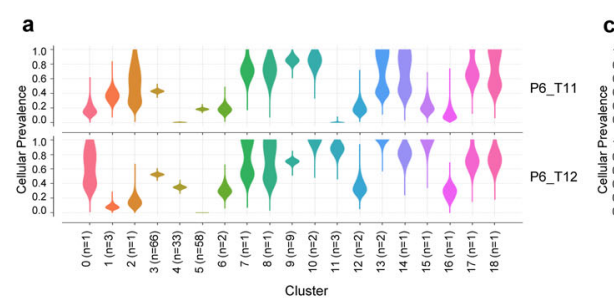

b

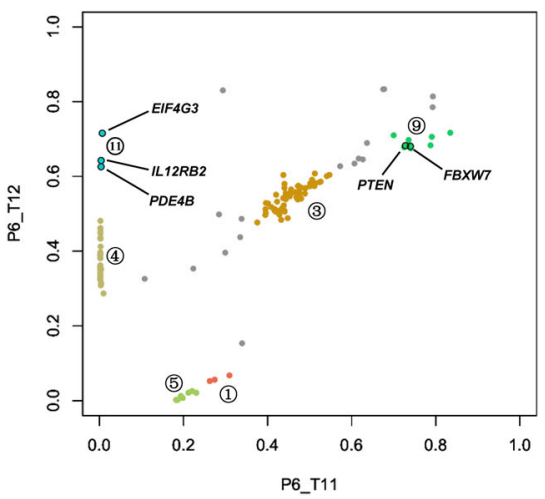

d

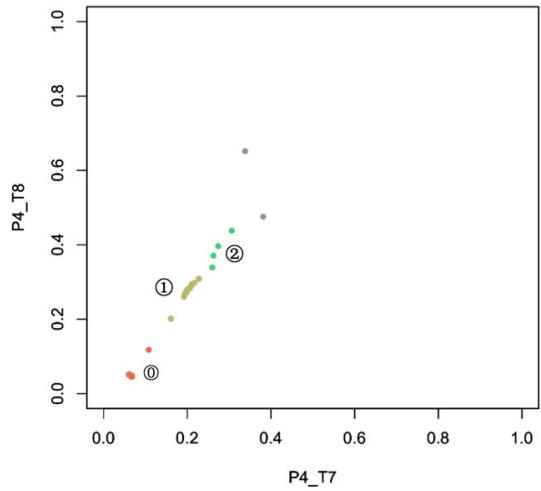

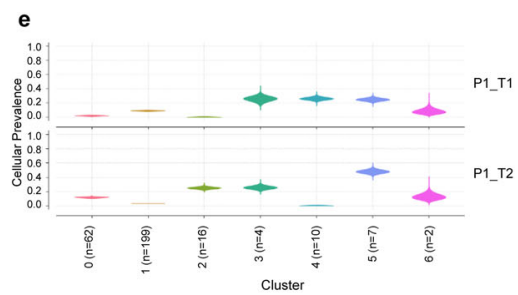

f $\mathbf{P 1}$

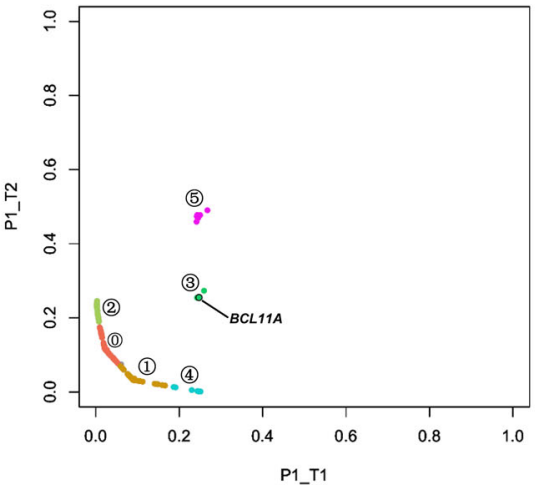

Fig. 3 Cancer cell fractions (CCFs) of subclones and mutations for patients P6, P4 and P1. We computed the CCF for each mutation found in the tumor samples from patients P6, P4, and P1. Hierarchical clustering of mutations is then performed to obtain putative subclones. a, c, e Subclone CCF density figure shown as violin plots. The $x$-axis represents subclone clusters, and the $\mathrm{n}$ value shows the number of mutations in each subclone. Each mutation has two CCF values (as they may be detected in both tumor samples). The $y$-axis represents the density of CCFs for each subclone. $\mathbf{b}, \mathbf{d}, \mathbf{f}$ Scatter plot of CCFs for each patient. Each point represents a mutation. The $x$ - and $y$-axis represent the CCFs of a mutation for each of two tumor samples in a patient. Mutations belonging to clusters $n \geq 3$ are shown in their corresponding cluster colors in the violin plot. Mutations belonging to clusters $n<3$ are outliers (shown in light grey) and not clustered. a 19 PyClone inferred subclone clusters in P6. $\mathbf{b}$ CCF relationship for P6. Subclone 1, 3, 4, 5, 9, and 11 in a are marked. PTEN, FBXW7, EIF4G3, IL12RB2, and PDE4B mutations are labelled with arrows. c Five PyClone inferred subclone clusters in P4. d CCF relationship for P4. Subclone 0, 1, and 2 in c are marked. e Seven PyClone inferred subclone clusters in P1. f CCF relationship for P1. Subclone $0,1,2,3,4$, and 5 in e are marked. BCL11A mutation is labelled with arrows.

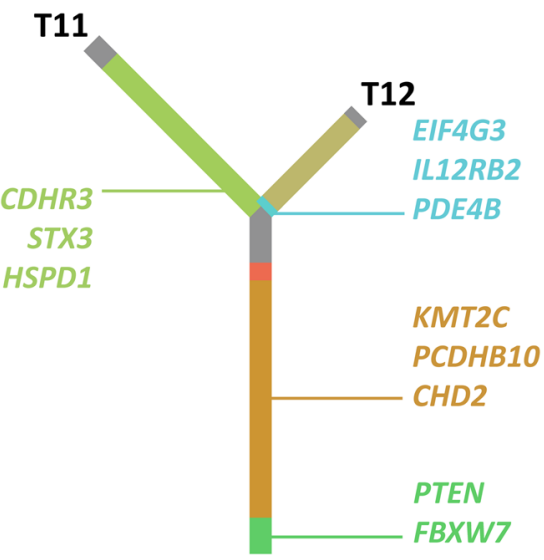

P6

Fig. 4 The Phylogenetic tree of tumor samples from patient P6. The trunk of the tree represents clusters of mutations that are common to both tumor samples, likely representing truncal mutations. The lengths of the trunk and the branches of the tree are proportional to the number of corresponding mutations. Major subclones are labelled in the same color as in Fig. 3b. Gene labels refer to mutations in genes that are identified in T11 only, T12 only, or common to both tumors. The gene labels are nonexhaustive.

all located in chromosome 1. Figure 4 shows the phylogenetic tree for P6. In order to further explore the relationships between different subclones in patient P6, we constructed the subclonal architecture based on cluster CCFs (see Methods). Supplementary
Figure 3 depicts the deduced linear and/or branching relationships of subclones in P6. For example, in architecture c (one of the four possible subclonal architectures of sample T11), subclone 9 represented the subclonal trunk mutations, with subclone 3, 1, and 5 all derived from it (i.e., they were all linear in relationship to subclone 9). Subclone 5 was derived from subclone 3, but subclone 3 and 1 occupied different subpopulations of cells (i.e., subclones 3 and 1 were diverging branches).

Three major subclones were found in patient P4 (Fig. 3c, d), and their subclone CCFs had little differences between the two samples, indicating high similarity between tumor samples from P4.

Six major subclones were identified in patient P1 (Fig. 3e, f). Most of the mutations had CCFs below 0.2 (subclone 0 and 1), while subclone 2 and 4 reflected ITH. Also, a mutation of the driver gene $B C L 11 A^{28}$ was found in subclone 3 . Figure $1 \mathrm{~b}$ shows these important functional genes.

\section{DISCUSSION}

To obtain a better understanding of the genomic alterations and ITH in inflammatory breast cancer, we applied WES to matched normal and tumor samples of IBC patients. Herein, we report the frequently mutated genes, varying levels of $\mathrm{ITH}$, subclonal structures and possible driver genes in different patients. Our study is one of the few attempts using WES to analyze IBC ${ }^{19}$ and investigate ITH with subclonal structures in IBC.

Previous studies have reported the proportion of positive receptors in IBC tumors. The prevalence of overexpressed or amplified HER2 was about $40 \%$ (compared with $25 \%$ in non-IBCs), and the prevalence of HR positivity is lower, about 30\% (compared with $60-80 \%$ in non-IBCs) ${ }^{29}$. The HR+ percentage of IBC tumors in recent NGS-based studies was about 39\% (ranging from 29 to 
$54 \%)^{12-18}$. However, since HR+ IBC patients tend to have worse clinical outcomes than $\mathrm{HR}+$ non-IBC patients ${ }^{29}$, this study sought to explore the genomic landscape of HR+ IBC tumors. This strategy also prevents potential confounding effects from HR subtypes, in contrast to previous IBC studies.

We found a frequently mutated gene $K M T 2 C$, which has been reported as frequently altered in other $\mathrm{IBC}^{30}$ (15\% mutation rate) and non-IBC ${ }^{16}$ cases ( $11 \%$ mutation rate). As a reported driver gene, KMT2C had the highest genetic mutation rate among histone methyltransferases in breast cancer and was most frequently mutated in Luminal A breast cancer ${ }^{31}$. Previous works demonstrated that KMT2C mediated ER-independent growth of $\mathrm{HR}+$ breast cancer cell lines ${ }^{24,32}$ and KMT2C loss promoted hormone-independent ER+ breast cancer cell proliferation ${ }^{32}$. Thus, the HR positivity of our samples could be an important factor for the enrichment of KMT2C mutation found in our study (a $42 \%$ mutation rate). The deletion of $K M T 2 C$ is significantly associated with shorter PFS ${ }^{32}$, and amplification/gain of this gene was significantly associated with longer survival, compared with patients who had no change in copy number ${ }^{32}$.

In patient P6, PTEN and FBXW7 mutations were detected at high CCFs, thus they may be driver mutations for this patient. The lipid phosphatase PTEN is a major negative regulator of the PI3K/Akt/ mammalian target of rapamycin (mTOR) pathway ${ }^{26}$. PI3K inhibitors, such as alpelisib, have been approved for treatment of PIK3CA-mutant ER + breast cancers ${ }^{33}$. Everolimus (a rapamycin analog and an inhibitor of the mTOR pathway) has also been approved for ER+ breast cancer ${ }^{34}$. FBXW7 is a critical tumor suppressor, which controls the proteasome-mediated degradation of $\mathrm{mTOR}^{27}$. Human breast cancer cell lines harboring deletions or mutations in FBXW7 are particularly sensitive to rapamycin treatment ${ }^{27}$. Finally, breast cancer patients with lower FBXW7 mRNA expression had poorer survival ${ }^{35}$.

Also in patient P6, EIF4G3, IL12RB2, and PDE4B mutations only occurred in sample $\mathrm{T} 12$ and formed a subclone with relatively high CCF (>0.6). This was an interesting finding as it indicated that this subclone was newly generated only in a specific area of the tumor. These genes seemed to have a strong positive selection in specific environment and conditions, as well as a potential to drive secondary tumor progression.

Phosphodiesterase type IV (PDE4) degrades the intracellular second messenger cyclic AMP in many cell types. As PDE4s regulate many active processes such as immune cell proliferation and inflammatory mediators releasing, PDE4 inhibitors are potent inhibitors of inflammation, and they have been approved for the treatment of many inflammatory diseases including asthma, arthritis and chronic obstructive pulmonary disease ${ }^{36,37}$. Previous works showed that $P D E 4 B$ is a potential therapeutic target as well as prognostic molecular marker in colorectal cancer ${ }^{38,39}$. Further study is needed to investigate if $P D E 4 B$ could also be a therapeutic target or marker for IBC patients.

IL12RB2, which encodes for one chain of the interleukin-12 (IL12) receptor, is involved in several inflammatory diseases ${ }^{40}$. IL-12 is a heterodimeric proinflammatory cytokine. Overexpression of IL12 can cause persistent inflammation ${ }^{41}$, thus contributing to the aggressive nature of $\mathrm{IBC}^{29}$. Genetic polymorphisms in IL12RB2 are associated with increased risk of chronic inflammatory disease ${ }^{42}$. Also, hyperactivation of the IL-6 pathway is frequently observed in IBC, and associated with poor prognosis ${ }^{29}$. In our samples, we observed a high percentage of tumor cells harboring IL12RB2 mutations (i.e., high CCF), though it remains unclear whether the IL12RB2 mutations play any functional roles in influencing the inflammatory pathways.

The presence of ITH in patients with IBC or other cancers indicates that an individual tissue biopsy may be insufficient to evaluate the genomic profile of an entire tumor, which could introduce bias in the selection of personalized therapies. For example, the gene coding for the estrogen receptor, ESR1, is often found to be mutated in metastatic ER+ breast cancers previously treated with estrogen therapy ${ }^{43}$. The high ESR1 mutational prevalence in previously treated tumors, juxtaposed with the rarity of ESR1 mutations in treatment-naïve primary tumors, suggest the development of resistance subclones during treatment, and thus has raised much interest in understanding $1 \mathrm{TH}^{43}$. Furthermore, several landmarks of disease progression in breast cancer, such as resistance to chemotherapy and metastases, arose within detectable subclones in the primary tumor ${ }^{44}$. These findings highlight the importance of subclonal structure analysis.

In this study, conducting WES on multiple samples from each IBC tumor allowed us to investigate many more genes than using targeted sequencing, and thus we were able to identify specific subclonal structures and ITH. However, the main limitation of our study is the small sample size. Given the rarity of IBC, many genomic studies on this disease subtype face challenges in acquiring enough samples. In this study, the tumor tissues without matched normal specimens further reduced the number of available samples. Moreover, although we demonstrated extensive ITH in HR+ IBC, the limited sample size prevented us from reaching more definitive conclusions on the role of clonal expansion in IBC. One interesting aspect is the genomic level comparison between $\mathrm{IBCs}$ and non-IBCs, which remains underexplored. A previous study using immunohistochemistry suggested overexpression of E-cadherin to be a key difference ${ }^{45}$, but large-scale nonbiased approaches are also needed. Further research comparing IBC and non-IBC samples with matched clinical characteristics may uncover the genomic origin of IBC. To definitively answer the effects of clonal expansion on the inflammatory phenotype of IBC, non-IBC patients who have inflammatory recurrence during follow-up could be enrolled, to compare primary non-IBC tumor tissues with tumor tissue at recurrence. Another limitation of this study is the lack of information regarding treatments prior to sample collection for some patients. Patient P6 received chemotherapy before sample collection, which could possibly influence the genomic signature and result in significant ITH.

In conclusion, we conducted WES on multiple samples of human IBC tumors with matched normal samples, and our results revealed the high frequency and diversity of somatic mutations, subclonal structures, differing levels of ITH, and potential driver genes in IBC patients. These findings encourage future studies and clinical trials for developing targeted therapies that could benefit IBC patients.

\section{METHODS}

\section{Patient samples}

Sixteen samples were collected from six IBC patients, including 12 tumors (two from each patient) and 4 matched normal samples (in four out of six patients). The six patients P1-P6 were enrolled between 1993 and 2012. This study was based on detecting archived tissue samples and reviewing archived medical/pathologic reports. Patient consent was waived by the Institutional Review Board of the Office of Human Research at Thomas Jefferson University under an approved protocol.

\section{Identification of molecular subtype}

Immunohistochemical (IHC) staining of paraffin-embedded tissue sections with monoclonal antibodies were used to determine patients' ER and PR status as part of a routine diagnostic procedure. HR status was positive if the patients were either ER or PR positive. HER2 status was also determined by $\mathrm{IHC}$ staining following standard guidelines at the time of diagnosis. The FDA approved DAKO guidelines were used for scoring patient P5 $(2004)^{46,47}$. The 2007 ASCO/CAP guideline ${ }^{48}$ was used for patient P6 (2012). There were no standard guidelines before the FDA approval, therefore we matched the old scoring systems ${ }^{49,50}$ with modern standards for those early patients (P1-P4). The percentage of ER- and PR-positive cells and HER2 status scores were obtained from pathological reports and shown in Supplementary Table 1. 


\section{DNA extraction and WES}

For all tumor samples, IBC diagnosis was confirmed by two independent pathologists and the tumor regions were macro-dissected under a microscope. For each sample, we extracted total DNA from approximately ten 14-um sections of formalin-fixed, paraffin-embedded (FFPE) blocks (tissue surface area, $100-150 \mathrm{~mm}^{2}$ ) using the AllPrep DNA/RNA FFPE kit (Qiagen), with a protocol we empirically optimized. The AllPrep kit is wellvalidated on long-term preserved FFPE samples ${ }^{51,52}$. Before library construction, all DNA samples were assessed using a NanoDrop spectrophotometer for OD 260/280 and OD 260/230, a Qubit fluorometer for concentration, and a 2100 Bioanalyzer (Agilent) for peak analysis. We then performed WES (using SeqCap EZ Exome 2.0 kit from Nimblegen for library construction) on Illumina HiSeq 2000 paired-end sequencing system.

The human genome GRCh37 was used as a reference and the raw reads were aligned using BWA-0.7.17 $7^{53}$. The BAM files were generated through samtools-1.9, then further processed through duplicates marking, Base Quality Score Recalibration (BQSR), gVCF generating, joint genotyping and Variant Quality Score Recalibration (VQSR) by GATK-4.1.0.0 $0^{54}$. The sequencing quality assessment was evaluated by QPLOT ${ }^{55}$.

\section{Mutation calling and quality control}

Based on the best practice procedures for sequencing alignment and quality control ${ }^{56}$, somatic mutations were called by MuTect2 using genomic references from the Broad Institute ${ }^{57}$. We created a Panel of Normals (PoN) by aggregating all the normal samples so that we could remove common germline variants as well as commonly noisy sites (e.g., mapping artifacts or other somewhat random but systematic artifacts of sequencing). This PoN also served as the normal sample for P3 and P5 since they did not have matched normal samples for somatic calling. We applied the default filter to conservatively select somatic calls with confidence.

Final mutation calls were selected through a stringent filtering process and functionally annotated by ANNOVAR ${ }^{58}$.

We applied the following filtering criteria for somatic mutation calling: (1) read depth $>25$; (2) mutant $\mathrm{AF}>0.05$ in tumor samples; (3) corresponding allele frequency $<0.01$ in matched normal samples (if present); (4) mutations listed in 1000 Genomes Project ${ }^{59}$ or Exome Sequencing Project ${ }^{60}$ removed.

The following filtering criteria were applied for germline variant calling: (1) read depth $\geq 50$; (2) genotype quality score $\geq 30$; (3) allele fractions $\geq 0.3$ and $\leq 0.7$; (4) multiple-allele variants removed; (5) variant quality score recalibration $(\mathrm{VQSR}) \leq 97.00$; (6) variants in segmental duplication removed $^{61}$.

We validated the quality of our somatic mutation calls using methods that we have previously established ${ }^{61}$. Briefly, when running Mutect2 in patients with matched normal samples (P1, P2, P4, and P6), we performed the same pipeline and filtering criteria but switched the normal and tumor samples. The mutation calls that passed the criteria are declared as artifactual mutations. If there were major artifacts in FFPE samples, we would be able to call artifactual mutations in matched normal samples since they were also FFPE samples.

\section{Copy number aberration (CNA) inference}

CNAs were inferred using TITAN-1.26.0 25 based on the called germline heterozygous variants information. CNA analysis was only performed on tumor samples with matched normal.

First, we used HMMcopy- $0.99 .0^{62}$ to count the number of reads in nonoverlapping windows of $10 \mathrm{~kb}$ directly from BAM files. Then we obtained corrected read depth using mappability and GC content. CNAs were inferred by the ratios of tumor/normal, mutant/reference depths at the germline heterozygous variants sites. We set the maximum copy number to 5 and the number of clonal clusters to 2 in the TITAN settings.

\section{Subclone inference}

Finally, we inferred subclones using PyClone- $0.13 .1^{63}$ based on the obtained CNA information. PyClone is a hierarchical Bayes statistical model that uses the measurement of allelic prevalence in deep sequencing data to estimate the proportion of tumor cells harboring a mutation (referred to herein as 'cancer cell fraction' (CCF) ${ }^{63}$. We first computed the CCF for each mutation, and then performed hierarchical clustering to assign each mutation to one cluster (subclone).

In the PyClone settings, the number of iterations was set to 50,000 and the density model was chosen to be Beta Binomial emission. In order to obtain a better result, we optimized the input parameters and custom-built the yaml mutations files.

\section{Construction of subclonal architecture}

We deduced linear and/or branching evolutionary relationships of all subclones in patient P6 based on their cluster CCFs using established methods $^{61}$. A linear relationship between two subclones would indicate that the one with smaller CCF was derived from the one with larger CCF, suggesting that the mutations in the derived subclone occurred later in the same ancestral cells, which already carried the mutations in the larger subclone. A branching relationship between two subclones would indicate that the mutations in each of the subclones occurred in different ancestral cells and the subclones occupied different portions of the tumor cells.

\section{Reporting summary}

Further information on research design is available in the Nature Research Reporting Summary linked to this article.

\section{DATA AVAILABILITY}

The data generated and analyzed during this study are described in the following data record: https://doi.org/10.6084/m9.figshare. $14538252^{64}$. Release of full genetic sequencing data was not included in the IRB protocol. Thus, only sequencing data related to this paper have been released, and these data have been deposited in NCBI Sequence Read Archive (SRA) with the accession code https://identifiers.org/ ncbi/bioproject:PRJNA71335965. Additional files underlying the figures and supplementary figures are available as part of the figshare data record.

\section{CODE AVAILABILITY}

The codes for Fig. 1 and Fig. 3, which were written in Python and R languages are available upon request.

Received: 23 September 2020; Accepted: 11 May 2021; Published online: 01 June 2021

\section{REFERENCES}

1. Ellis, D. L. \& Teitelbaum, S. L. Inflammatory carcinoma of the breast. A pathologic definition. Cancer 33, 1045-1047 (1974).

2. Jaiyesimi, I. A., Buzdar, A. U. \& Hortobagyi, G. Inflammatory breast cancer: a review. J. Clin. Oncol. 10, 1014-1024 (1992).

3. Robertson, F. M. et al. Inflammatory breast cancer: the disease, the biology, the treatment. CA Cancer J. Clin. 60, 351-375 (2010).

4. Anderson, W. F., Schairer, C., Chen, B. E., Hance, K. W. \& Levine, P. H. Epidemiology of inflammatory breast cancer (IBC) 1. Breast Dis. 22, 9-23 (2006).

5. Levine, P. H., Steinhorn, S. C., Ries, L. G. \& Aron, J. L. Inflammatory breast cancer: the experience of the surveillance, epidemiology, and end results (SEER) program. J. Natl Cancer Inst. 74, 291-297 (1985).

6. Hance, K. W., Anderson, W. F., Devesa, S. S., Young, H. A. \& Levine, P. H. Trends in inflammatory breast carcinoma incidence and survival: the surveillance, epidemiology, and end results program at the National Cancer Institute. J. Natl Cancer Inst. 97, 966-975 (2005).

7. Brewer, T. et al. Statin use in primary inflammatory breast cancer: a cohort study. Br. J. Cancer 109, 318-324 (2013).

8. Chang, S., Parker, S. L., Pham, T., Buzdar, A. U. \& Hursting, S. D. Inflammatory breast carcinoma incidence and survival: The Surveillance, Epidemiology, and End Results program of the National Cancer Institute, 1975-1992. Cancer Interdiscip. Int. J. Am. Cancer. Soc. 82, 2366-2372 (1998).

9. Tabassum, D. P. \& Polyak, K. Tumorigenesis: it takes a village. Nat. Rev. Cancer 15 , 473-483 (2015).

10. Stanta, G. \& Bonin, S. Overview on clinical relevance of intra-tumor heterogeneity. Front. Med. 5, 85 (2018).

11. Neelakantan, D., Drasin, D. J. \& Ford, H. L. Intratumoral heterogeneity: clonal cooperation in epithelial-to-mesenchymal transition and metastasis. Cell Adhes. Migr. 9, 265-276 (2015).

12. Matsuda, N. et al. Identification of frequent somatic mutations in inflammatory breast cancer. Breast Cancer Res. Treat. 163, 263-272 (2017).

13. Ross, J. S. et al. Comprehensive genomic profiling of inflammatory breast cancer cases reveals a high frequency of clinically relevant genomic alterations. Breast Cancer Res. Treat. 154, 155-162 (2015). 
14. Liang, $X$. et al. Targeted next-generation sequencing identifies clinically relevant somatic mutations in a large cohort of inflammatory breast cancer. Breast Cancer Res. 20, 1-12 (2018).

15. Hamm, C. A. et al. Genomic and immunological tumor profiling identifies targetable pathways and extensive CD8+/PDL1+ immune infiltration in inflammatory breast cancer tumors. Mol. Cancer Ther. 15, 1746-1756 (2016).

16. Bertucci, F. et al. NOTCH and DNA repair pathways are more frequently targeted by genomic alterations in inflammatory than in non-inflammatory breast cancers. Mol. Oncol. 14, 504-519 (2020).

17. Winn, J. S. et al. Genetic variants detected using cell-free DNA from blood and tumor samples in patients with inflammatory breast cancer. Int. J. Mol. Sci. 21, 1290 (2020).

18. Bingham, C. et al. Mutational studies on single circulating tumor cells isolated from the blood of inflammatory breast cancer patients. Breast Cancer Res. Treat. 163, 219-230 (2017).

19. Goh, G. et al. Clonal evolutionary analysis during HER2 blockade in HER2-positive inflammatory breast cancer: a phase II open-label clinical trial of afatinib +/-vinorelbine. PLoS Med. 13, e1002136 (2016).

20. Doebar, S. et al. Progression of ductal carcinoma in situ to invasive breast cancer: comparative genomic sequencing. Virchows Arch. 474, 247-251 (2019).

21. Alexandrov, L. B. et al. Signatures of mutational processes in human cancer. Nature 500, 415-421 (2013).

22. Nik-Zainal, S. et al. Landscape of somatic mutations in 560 breast cancer wholegenome sequences. Nature 534, 47-54 (2016).

23. Pereira, B. et al. The somatic mutation profiles of 2,433 breast cancers refine their genomic and transcriptomic landscapes. Nat. Commun. 7, 1-16 (2016).

24. Bertucci, F. et al. Genomic characterization of metastatic breast cancers. Nature 569, 560-564 (2019).

25. Ha, G. et al. TITAN: inference of copy number architectures in clonal cell populations from tumor whole-genome sequence data. Genome Res. 24, 1881-1893 (2014).

26. Chalhoub, N. \& Baker, S. J. PTEN and the PI3-kinase pathway in cancer. Annu. Rev. Pathol. 4, 127-150 (2009)

27. Mao, J.-H. et al. FBXW7 targets mTOR for degradation and cooperates with PTEN in tumor suppression. Science 321, 1499-1502 (2008).

28. Khaled, W. T. et al. BCL11A is a triple-negative breast cancer gene with critical functions in stem and progenitor cells. Nat. Commun. 6, 1-10 (2015).

29. Lim, B., Woodward, W. A., Wang, X., Reuben, J. M. \& Ueno, N. T. Inflammatory breast cancer biology: the tumour microenvironment is key. Nat. Rev. Cancer 18, 485-499 (2018).

30. Cheasley, D. et al. Molecular comparison of interval and screen-detected breast cancers. J. Pathol. 248, 243-252 (2019).

31. Liu, L., Kimball, S., Liu, H., Holowatyj, A. \& Yang, Z.-Q. Genetic alterations of histone lysine methyltransferases and their significance in breast cancer. Oncotarget $\mathbf{6}$, 2466 (2015).

32. Gala, K. et al. KMT2C mediates the estrogen dependence of breast cancer through regulation of ERa enhancer function. Oncogene 37, 4692-4710 (2018).

33. André, F. et al. Alpelisib for PIK3CA-mutated, hormone receptor-positive advanced breast cancer. N. Engl. J. Med. 380, 1929-1940 (2019).

34. Hidalgo, M. \& Rowinsky, E. K. The rapamycin-sensitive signal transduction pathway as a target for cancer therapy. Oncogene 19, 6680-6686 (2000).

35. Ibusuki, M., Yamamoto, Y., Shinriki, S., Ando, Y. \& Iwase, H. Reduced expression of ubiquitin ligase FBXW7 mRNA is associated with poor prognosis in breast cancer patients. Cancer Sci. 102, 439-445 (2011).

36. Manning, C. D. et al. Suppression of human inflammatory cell function by subtype-selective PDE4 inhibitors correlates with inhibition of PDE4A and PDE4B. $\mathrm{Br}$ J. Pharmacol. 128, 1393-1398 (1999).

37. Lai, S. H., Zervoudakis, G., Chou, J., Gurney, M. E. \& Quesnelle, K. M. PDE4 subtypes in cancer. Oncogene 39, 3791-3802 (2020).

38. Kim, D. U., Kwak, B. \& Kim, S.-W. Phosphodiesterase 4B is an effective therapeutic target in colorectal cancer. Biochem. Biophys. Res. Commun. 508, 825-831 (2019).

39. Tsunoda, T. et al. Inhibition of phosphodiesterase-4 (PDE4) activity triggers luminal apoptosis and AKT dephosphorylation in a 3-D colonic-crypt model. Mol. Cancer 11, 1-12 (2012)

40. Mizuki, N. et al. Genome-wide association studies identify IL23R-IL12RB2 and IL10 as Behçet's disease susceptibility loci. Nat. Genet. 42, 703-706 (2010).

41. Gee, K., Guzzo, C., Che Mat, N. F., Ma, W. \& Kumar, A. The IL-12 family of cytokines in infection, inflammation and autoimmune disorders. Inflamm. Allergy Drug Targets 8, 40-52 (2009).

42. Chognard, G. et al. The dichotomous pattern of IL-12r and IL-23R expression elucidates the role of IL-12 and IL-23 in inflammation. PLOS ONE 9, e89092 (2014).

43. Reinert, T., Saad, E. D., Barrios, C. H. \& Bines, J. Clinical implications of ESR1 mutations in hormone receptor-positive advanced breast cancer. Front. Oncol. 7, 26 (2017).

44. Yates, L. R. et al. Subclonal diversification of primary breast cancer revealed by multiregion sequencing. Nat. Med. 21, 751-759 (2015).
45. Kleer, C. G., van Golen, K. L., Braun, T. \& Merajver, S. D. Persistent E-cadherin expression in inflammatory breast cancer. Mod. Pathol. 14, 458-464 (2001).

46. Varga, Z., Noske, A., Ramach, C., Padberg, B. \& Moch, H. Assessment of HER2 status in breast cancer: overall positivity rate and accuracy by fluorescence in situ hybridization and immunohistochemistry in a single institution over 12 years: a quality control study. BMC Cancer 13, 615 (2013).

47. Lebeau, A. et al. Her-2/neu analysis in archival tissue samples of human breast cancer: comparison of immunohistochemistry and fluorescence in situ hybridization. J. Clin. Oncol. 19, 354-363 (2001).

48. Wolff, A. C. et al. American Society of Clinical Oncology/College of American Pathologists guideline recommendations for human epidermal growth factor receptor 2 testing in breast cancer. Arch. Pathol. Lab Med. 131, 18-43 (2007).

49. Slamon, D. J. et al. Studies of the HER-2/neu proto-oncogene in human breast and ovarian cancer. Science 244, 707-712 (1989).

50. Cobleigh, M. A. et al. Multinational study of the efficacy and safety of humanized anti-HER2 monoclonal antibody in women who have HER2-overexpressing metastatic breast cancer that has progressed after chemotherapy for metastatic disease. J. Clin. Oncol. 17, 2639-2648 (1999).

51. Ludyga, N. et al. Nucleic acids from long-term preserved FFPE tissues are suitable for downstream analyses. Virchows Arch. 460, 131-140 (2012).

52. Patel, P. G. et al. Reliability and performance of commercial RNA and DNA extraction kits for FFPE tissue cores. PLOS ONE 12, e0179732 (2017).

53. Li, H. \& Durbin, R. Fast and accurate short read alignment with Burrows-Wheeler transform. Bioinformatics 25, 1754-1760 (2009).

54. McKenna, A. et al. The Genome Analysis Toolkit: a MapReduce framework for analyzing next-generation DNA sequencing data. Genome Res. 20, 1297-1303 (2010).

55. Li, B. et al. QPLOT: a quality assessment tool for next generation sequencing data BioMed Res. Int. 2013, 865181 (2013).

56. Van der Auwera, G. A. et al. From FastQ data to high-confidence variant calls: the genome analysis toolkit best practices pipeline. Curr. Protoc. Bioinformatics 43 11.10.1-11.10.33 (2013).

57. Cibulskis, K. et al. Sensitive detection of somatic point mutations in impure and heterogeneous cancer samples. Nat. Biotechnol. 31, 213-219 (2013).

58. Wang, K., Li, M. \& Hakonarson, H. ANNOVAR: functional annotation of genetic variants from high-throughput sequencing data. Nucleic Acids Res. 38, e164-e164 (2010).

59. Consortium, G. P. A global reference for human genetic variation. Nature $\mathbf{5 2 6}$ 68-74 (2015).

60. Fu, W. et al. Analysis of 6,515 exomes reveals the recent origin of most human protein-coding variants. Nature 493, 216-220 (2013).

61. Wei, Q. et al. Multiregion whole-exome sequencing of matched primary and metastatic tumors revealed genomic heterogeneity and suggested polyclonal seeding in colorectal cancer metastasis. Ann. Oncol. 28, 2135-2141 (2017).

62. Lai, D. \& Shah, S. HMMcopy: Copy number prediction with correction for GC and mappability bias for HTS data. https://rdrr.io/bioc/HMMcopy/ (2012).

63. Roth, A. et al. PyClone: statistical inference of clonal population structure in cancer. Nat. Methods 11, 396-398 (2014).

64. Luo, R. et al. Metadata record for the article: Whole-exome sequencing identifies somatic mutations and intratumor heterogeneity in inflammatory breast cancer. figshare https://doi.org/10.6084/m9.figshare.14538252 (2021).

65. Rui Luo. Sequence Read Archive https://identifiers.org/ncbi/bioproject:PRJNA713359 (2021).

\section{ACKNOWLEDGEMENTS}

This study was funded by The Jamie Lieberman Memorial Endowment Fund and The Inflammatory Breast Cancer Network Foundation, and in part by the National Cancer Institute Grant (R01CA207468) and the Pennsylvania Department of Health Grant (SAP\# 4100062221). Research reported in this publication utilized the Circulating Tumor Cell Core Facility at the Sidney Kimmel Cancer Center at Jefferson Health and was supported by the National Cancer Institute of the National Institutes of Health under Award Number P30CA056036. The funding agencies were not involved in the design, conduct, analysis, or interpretation of the study. Publication made possible in part by support from the Thomas Jefferson University Open Access Fund.

\section{AUTHOR CONTRIBUTIONS}

R.L., W.C., and H.Y. conceived the study. Z.Z. and Z.Y. prepared tissue samples. R.T.S. and W.J. provided patient clinical information and pathology reports. R.L., Q.W., and W.C. performed bioinformatics analysis. M.M.A., D.P.S., B.L., R.E.M., and M.C. reviewed all analyzed data. R.L., W.C., and C.W. prepared the manuscript. All authors discussed the results, revised, and approved the paper. R.L. and W.C. contributed equally to this study. 


\section{COMPETING INTERESTS}

M.M.A. received honorarium for a consultant/advisory role from AstraZeneca, Immunomedics, PUMA, Biothera, Biotheranostics, Agendia, Norvartis, and Lilly. M.C. received honorarium from Lilly, Menarini, Foundation Medicine, CytoDyn, G1 Therapeutics, and Sermonix. H.Y. is on the SAB of Oriomics Inc., a shareholder of Illumina, Pfizer, and Oriomics, and serves as a NIH study section reviewer. The above reported activities were not related to the research reported in this article. The remaining authors declare no competing interests.

\section{ADDITIONAL INFORMATION}

Supplementary information The online version contains supplementary material available at https://doi.org/10.1038/s41523-021-00278-w.

Correspondence and requests for materials should be addressed to H.Y.

Reprints and permission information is available at http://www.nature.com/ reprints
Publisher's note Springer Nature remains neutral with regard to jurisdictional claims in published maps and institutional affiliations.

(c) (i)

Open Access This article is licensed under a Creative Commons Attribution 4.0 International License, which permits use, sharing, adaptation, distribution and reproduction in any medium or format, as long as you give appropriate credit to the original author(s) and the source, provide a link to the Creative Commons license, and indicate if changes were made. The images or other third party material in this article are included in the article's Creative Commons license, unless indicated otherwise in a credit line to the material. If material is not included in the article's Creative Commons license and your intended use is not permitted by statutory regulation or exceeds the permitted use, you will need to obtain permission directly from the copyright holder. To view a copy of this license, visit http://creativecommons. org/licenses/by/4.0/.

(c) The Author(s) 2021 